\title{
High-Frequency Audiometry for Early Detection of Hearing Loss: A Narrative Review
}

\author{
Michaela Škerková ${ }^{1, * \mathbb{D}}$, Martina Kovalová ${ }^{1}$ and Eva Mrázková ${ }^{1,2,3}$ \\ 1 Department of Epidemiology and Public Health, Faculty of Medicine, University of Ostrava, \\ 70300 Ostrava, Czech Republic; martina.kovalova@osu.cz (M.K.); orl.centrum@centrum.cz (E.M.) \\ 2 Center for Hearing and Balance Disorders, 70800 Ostrava, Czech Republic \\ 3 Department of ENT, Regional Hospital Havírov, 73601 Haviŕov, Czech Republic \\ * Correspondence: michaela.skerkova@osu.cz; Tel.: +420-799-500-985
}

Citation: Škerková, M.; Kovalová, M.; Mrázková, E. High-Frequency Audiometry for Early Detection of Hearing Loss: A Narrative Review. Int. J. Environ. Res. Public Health 2021, 18, 4702. https://doi.org/10.3390/ ijerph18094702

Academic Editors: Martine Hamann and Yvonne De Kluizenaar

Received: 28 February 2021

Accepted: 24 April 2021

Published: 28 April 2021

Publisher's Note: MDPI stays neutral with regard to jurisdictional claims in published maps and institutional affiliations.

Copyright: (c) 2021 by the authors. Licensee MDPI, Basel, Switzerland. This article is an open access article distributed under the terms and conditions of the Creative Commons Attribution (CC BY) license (https:// creativecommons.org/licenses/by/ $4.0 /)$.

\begin{abstract}
The WHO considers hearing loss to be a major global problem. A literature search was conducted to see whether high-frequency audiometry (HFA) could be used for the early detection of hearing loss. A further aim was to see whether any differences exist in the hearing threshold using conventional audiometry (CA) and HFA in workers of different age groups exposed to workplace noise. Our search of electronic databases yielded a total of 5938 scientific papers. The inclusion criteria were the keywords "high frequency" and "audiometry" appearing anywhere in the article and the participation of unexposed people or a group exposed to workplace noise. Fifteen studies met these conditions; the sample size varied (51-645 people), and the age range of the people studied was 5-90 years. Commercial high-frequency audiometers and high-frequency headphones were used. In populations unexposed to workplace noise, significantly higher thresholds of $14-16 \mathrm{kHz}$ were found. In populations with exposure to workplace noise, significantly higher statistical thresholds were found for the exposed group (EG) compared with the control group (CG) at frequencies of 9-18 kHz, especially at $16 \mathrm{kHz}$. The studies also showed higher hearing thresholds of $10-16 \mathrm{kHz}$ in respondents aged under 31 years following the use of personal listening devices (PLDs) for longer than 5 years. The effect of noise-induced hearing loss (NIHL) first became apparent for HFA rather than CA. However, normative data have not yet been collected. Therefore, it is necessary to establish a uniform evaluation protocol accounting for age, sex, comorbidities and exposures, as well as for younger respondents using PLDs.
\end{abstract}

Keywords: audiometry; high-frequency audiometry (HFA); hearing loss; hearing test; hearing threshold; noise-induced hearing loss (NIHL); age-related hearing loss; occupational noise; recreational noise; noise exposure

\section{Introduction}

The monitoring of hearing functions in the context of the International Classification of Functioning, Disability, and Health has revealed that hearing loss is a major global problem [1]. The World Health Organization (WHO) speaks of hearing loss as an epidemic of the 21st century [2]. More than 1 billion young people (aged 12-35 years) are at risk of hearing loss due to recreational exposure to intense sound [3]. Hearing loss is an invisible and very stigmatizing chronic disease. In terms of severity, hearing loss is second only to mental disability. It is the most common and the most serious human sensory defect. The incidence of hearing loss is continuously increasing and, at the same time, as with other diseases, prevention and early detection of hearing loss can lead to earlier provision of appropriate care. This could significantly lower the incidence of damage and result in better quality of life [2,3]. In its 2017 report, the Lancet Commission on dementia prevention, intervention and care found that $35 \%$ of dementia cases are attributable to a combination of nine modifiable risk factors, divided into early-life, mid-life and late-life risk factors. The most significant among the mid-life (45-65 years of age) risk factors 
is hearing loss, as 55 is the mean age at which the presence of hearing loss is shown to increase the risk of dementia. Despite being classified as a mid-life risk factor, evidence suggests that it continues to increase the dementia risk in later life. Although the report makes it clear that the exact mechanism through which hearing loss affects dementia risk is not fully understood, the authors cite studies suggesting that hearing loss might either add to the cognitive load of a vulnerable brain, leading to changes in the brain, or lead to social disengagement or depression and accelerated brain atrophy, all of which could contribute to an accelerated cognitive decline. Moreover, given the importance of language in humans (a key contributor to the coevolution of social interaction and a larger brain size), hearing loss could lead to uniquely interrelated and detrimental social, cognitive and brain effects [4]. When not detected, affected individuals gradually disengage from society and suffer from the accompanying consequences, such as loss of employment, loss of interpersonal communication and the associated intensification of social isolation and dementia. According to the WHO, the global prevalence of hearing loss in 2018 was 466 million people. Of these, 432 million were adults with impaired hearing. This number continues to increase as the average age of the world's population increases. According to WHO projections, the number of people suffering from hearing loss is expected to reach 630 million by 2030 and 900 million by 2050 [2,3]. The auditory system gradually deteriorates with age, starting as early as the age of 40 . The WHO states that approximately one-third of the population above the age of 65 suffers from hearing loss. The WHO cites the hear-it report from the Hearing Loss Association of America showing a hearing loss prevalence of $30 \%$ in people aged over 65 years of age $[5,6]$. The same report found that almost all people over 80 years of age have some degree of hearing impairment. Worsened hearing in the elderly is mainly associated with a reduced ability to comprehend speech, especially in noisy environments or in places with background noise. People with impaired hearing realize that someone is talking to them, but they are unable to understand what is being said [7-9].

Conventional pure tone audiometry (CA) is currently one of the most widely used methods for the diagnosis of hearing impairment. CA operates over the $0.125-8 \mathrm{kHz}$ frequency range. High-frequency audiometry (HFA) is used to examine the hearing threshold in the frequency range of $8-20 \mathrm{kHz}$. These are the frequencies at which damage to the hearing threshold can first be observed [10]. HFA has been studied for several decades, but the lack of commercially available equipment and the standardization of calibration recommendations have long limited its use. Specially adapted audiometers capable of generating tones with frequencies of up to $20 \mathrm{kHz}$ are used for the test. Experimental studies have established rules and conditions for testing [11,12]. Over the last 20 years, the development of new methods and devices and the increased penetration of computers in both daily life and healthcare have enabled HFA to increasingly become the standard. The current applicable standard, "IEC 60645-1 Electroacoustics-Audiometric equipment, Part 1: Equipment for pure-tone and speech audiometry", specifies the general requirements for audiometers designed for use in determining hearing threshold levels relative to the standard reference threshold levels, established by means of psychoacoustic test methods and those designed to perform psychoacoustic tests using speech material. The objective of this standard is to ensure that (1) hearing tests in the frequency range of $0.125-16 \mathrm{kHz}$ conducted on a given human ear and performed with different pure-tone audiometers that comply with this standard give the same results; (2) the results obtained represent a valid comparison between the hearing of the ear tested and the reference threshold of hearing; and (3) the means for presenting speech material to a subject in a standardized manner are provided. This ensures that hearing tests using a specific speech signal and a specific manner of signal presentation, when performed with different audiometers that comply with this standard, give very similar results. Furthermore, the standard classifies audiometers according to the range of test signals they present, the mode of operation or their presumed primary application [13]. Conventional pure tone audiometry along with HFA and equipment (headphones) are used according to the standards of EN ISO 8253- 
1:2010 Acoustics—Audiometric test methods and EN ISO 266:1997 Acoustics—Preferred frequencies [14,15]. These standards were last reviewed and confirmed in 2018 and 2021. Therefore, these versions remain current. HFA is currently not used in common practice to determine the presence of hearing impairment [12]. Some experimental studies have used electrophysiological examinations to monitor the effect of hearing damage due to noise exposure. Close monitoring of the outer hair cell function when hearing thresholds are clinically normal could provide a timely measure of noise-induced hearing damage, especially for individuals with high levels of noise exposure [16].

In this study, a literature search was conducted to find out whether HFA can be used for early hearing loss detection. Furthermore, we set out to find out whether there are differences in the hearing threshold, as shown using CA and HFA, among different age groups between workers who are exposed to noise in their workplaces and unexposed people.

\section{Materials and Methods}

\subsection{Search Strategy}

The literature search was conducted from 15 November 2020 to 20 January 2021 in the PubMed, Scopus and Web of Science electronic databases. The search strategy combined key words related to HFA.

\subsection{Inclusion Criteria}

The criteria for the selection of publications were as follows:

- The keywords "high frequency" and "audiometry" should appear somewhere in the text of the article;

- The date of publication is between 2000 and 2020, in line with the time frame determined by the standards IEC 60645-1; 2017, EN ISO 8253-1:2010 and EN ISO 266:1997 [13-15];

- The study includes either an unexposed population or a group exposed to workplace noise;

- The results are evaluated with statistics;

- It is possible to compare the hearing thresholds for $\mathrm{CA}$ and at least 5 high frequencies in the range of $9-20 \mathrm{kHz}$;

- The study is written in either English, Portuguese or Spanish.

\subsection{Exclusion Criteria}

All duplicate articles were excluded, as well as studies with unclear exposure conditions. Conference papers, letters and book chapter categories were excluded. Articles concerning hearing loss in association with ototoxic drugs, tinnitus or diabetes were also excluded.

\section{Results}

The search yielded a total of 5938 scientific publications. A total of 5855 publications that did not directly relate to the examined issues were excluded. Finally, 83 articles were selected, of which 30 were duplicated in three databases. The remaining 53 articles concerned HFA, but not all of them were relevant to our search, as 26 of them concerned hearing loss in association with ototoxic drugs, tinnitus or diabetes. Of the remaining 27 articles, 15 papers that met all six conditions for inclusion in our study were selected. Figure 1 presents a step-by-step representation of our screening process.

Tables 1 and 2 summarize the characteristics of the 15 studies included. Studies were conducted in Brazil $(n=4)$, Iran $(n=2)$, Italy $(n=2)$, Spain $(n=1)$, Saudi Arabia $(n=1)$, India $(n=1)$, China $(n=1)$, Greece $(n=1)$ and the USA $(n=1)$, and there was also a systematical review comprising multiple countries around the world $(n=1)$.

In this review, the studies were evaluated by the following parameters: the number of involved participants; the differences in hearing thresholds between an unexposed population and a population exposed to noise; the correlation between hearing thresholds, assessed by HFA and age, especially focused on particular age groups; the difference in 
hearing thresholds if the age groups defined by the studies had non-identical age ranges; and the similarity in hearing thresholds if the groups had identical age ranges.

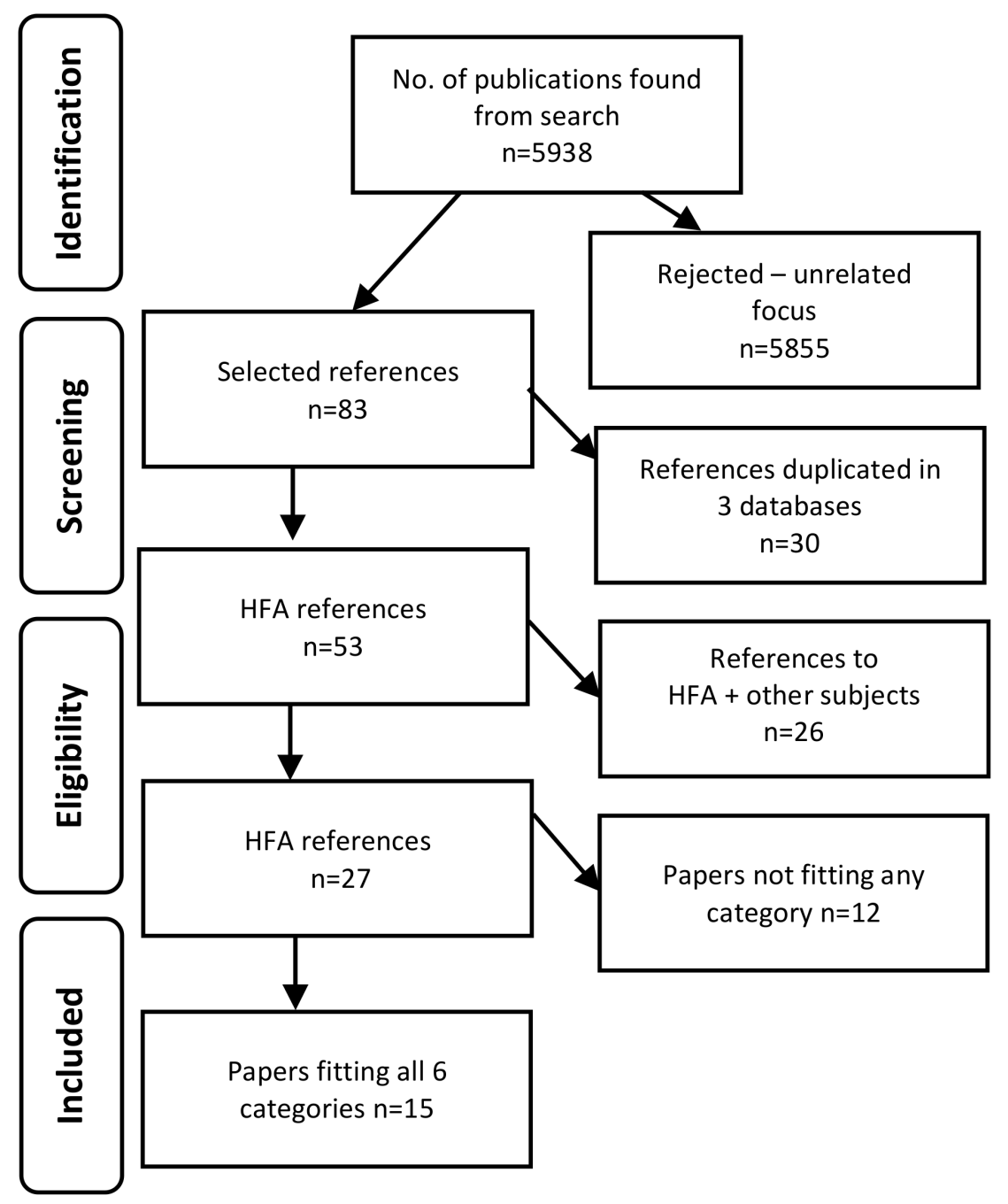

Figure 1. Flow chart of items used for the narrative review.

The number of participants in each study ranged from 51 to 645 . For the years 2000-2020, 5 articles were published in 2000-2010, 10 in 2011-2020, 5 in 2015-2020 and 2 in 2017-2020. The articles were divided into two groups. The first group comprised studies on people who were not exposed to workplace noise (Table 1). Two of these studies were focused on users of PLDs, one of the newly examined risk factors for hearing loss. The second group comprised studies on people who were exposed to noise in their workplace (EG), along with a control group (CG) without noise exposure (Table 2).

As shown in Table 1, the sample size varied from 51 to 645 participants in studies that were done on unexposed individuals. The age of the subjects varied from 5 to 90 years, depending on the study. Grouping based on age also varied, with all studies including both men and women. Rodríguez Valiente et al. studied 645 people who were evenly distributed among 10-year age groups ranging from 5 to 90 years, with 90 respondents in each group [17]. Other studies followed respondents in the age group of 18-31 years, with age intervals ranging from 11 to 15 years [18-20]. Oppitz et al. [21] used the largest age range. Kumar et al. and Le Prell et al. monitored the effect of personal listening devices (PLDs) [19,20]. Each study used a different type of audiometer [12-16]. Sennheiser headphones were used in four cases, and Rodríguez Valiente et al. and Le Prell used the Sennheiser HDA200 [17-20]. 
Table 1. Overview of the selected articles on hearing loss and high-frequency audiometry in populations not exposed to workplace noise.

\begin{tabular}{|c|c|c|c|c|c|c|c|c|}
\hline Author, Year & $\begin{array}{l}\text { Number of } \\
\text { Respondents and } \\
\text { Study Design }\end{array}$ & $\begin{array}{c}\text { Age Range (Years) and } \\
\text { Groups }\end{array}$ & $\begin{array}{l}\text { City, } \\
\text { Country }\end{array}$ & $\begin{array}{c}\text { Audiometry and } \\
\text { Frequency Range } \\
\text { (kHz) }\end{array}$ & $\begin{array}{l}\text { Audiometer } \\
\text { Type }\end{array}$ & Headphones & Objective & Findings \\
\hline $\begin{array}{l}\text { Rodríguez } \\
\text { Valiente et al., } \\
2014 \text { [17] }\end{array}$ & $\begin{array}{c}645 \\
\text { (321 men and } 324 \\
\text { women). No } \\
\text { workplace noise } \\
\text { exposure. } \\
\text { Prospective study. }\end{array}$ & $\begin{array}{c}5-90 \\
\text { Age groups: } 5-19,20-29, \\
\text { 30-39, 40-49, 50-59, } \\
60-69,70-90 . \\
\text { Same number in } \\
\text { each group. } \\
\text { Ears not distinguished. } \\
\text { Divided by gender. }\end{array}$ & $\begin{array}{l}\text { Madrid, } \\
\text { Spain }\end{array}$ & $\begin{array}{l}\text { CA } \\
0.125-8 \\
\text { HFA } \\
8-20\end{array}$ & $\begin{array}{l}\text { Madsen } \\
\text { Orbiter 922, } \\
\text { version 2, } \\
\text { Madsen } \\
\text { Electronics }\end{array}$ & $\begin{array}{c}\text { CA: } \\
\text { Telephonic } \\
\text { TDH-39P } \\
\text { HFA: } \\
\text { Sennheiser } 200\end{array}$ & $\begin{array}{l}\text { Determine threshold } \\
\text { values over the } \\
0.125-20 \mathrm{kHz} \text { range in } \\
\text { healthy, professional, } \\
\text { unexposed people; try to } \\
\text { set new standards. }\end{array}$ & $\begin{array}{c}\text { In the group of people aged } \\
20-69 \text { years, the hearing threshold } \\
\text { values were lower in women than } \\
\text { in men, especially at } 12.5 \text { and } \\
16 \mathrm{kHz} \text {. }\end{array}$ \\
\hline $\begin{array}{l}\text { Oppitz et al., } \\
2017 \text { [21] }\end{array}$ & $\begin{array}{c}60 \\
\text { (11 men and } \\
49 \text { women). } \\
\text { No workplace noise } \\
\text { exposure. } \\
\text { Cross-sectional, } \\
\text { prospective study. }\end{array}$ & $\begin{array}{l}18-58 \\
\text { Age groups: } 18-30,31-58 . \\
\text { Group 1: } 49 \text { people. } \\
\text { Group 2: } 11 \text { people. } \\
\text { Left and right ears. } \\
\text { Not divided by gender. }\end{array}$ & $\begin{array}{c}\text { Santa } \\
\text { Marie, Brazil }\end{array}$ & $\begin{array}{l}\text { CA } \\
0.250-8 \\
\text { HFA } \\
9-18\end{array}$ & $\begin{array}{c}\text { Interacoustics } \\
\text { AS10HF }\end{array}$ & $\begin{array}{l}\text { CA: } \\
\text { Telephonics } \\
\text { TDH-39P } \\
\text { HFA: } \\
\text { KOSS R/80 }\end{array}$ & $\begin{array}{l}\text { Evaluate high-frequency } \\
\text { hearing thresholds and } \\
\text { try to compare } \\
\text { differences between the } \\
\text { ears; verify correlation } \\
\text { between hearing quality } \\
\text { and aging. }\end{array}$ & $\begin{array}{l}\text { There was a progressive increase in } \\
\text { hearing thresholds above } 14 \mathrm{kHz} \text {. } \\
\text { The increased hearing thresholds } \\
\text { were found in both ears and were } \\
\text { proportional to the rising frequency } \\
\text { and age. }\end{array}$ \\
\hline $\begin{array}{c}\text { Barbosa de Sá } \\
\text { et al., } \\
2007 \text { [18] }\end{array}$ & $\begin{array}{c}51 \\
\text { (19 men and } \\
32 \text { women). } \\
\text { No workplace noise } \\
\text { exposure. } \\
\text { Cross-sectional, } \\
\text { prospective study. }\end{array}$ & $\begin{array}{l}\text { 18-29 } \\
1 \text { age group. } \\
\text { Left and right ears. } \\
\text { Divided by gender. }\end{array}$ & $\begin{array}{l}\text { Rio de } \\
\text { Janeiro, } \\
\text { Brazil }\end{array}$ & $\begin{array}{l}\text { CA } \\
0.250-8 \\
\text { HFA } \\
8-18\end{array}$ & Amplaid 460 & $\begin{array}{c}\text { CA: } \\
\text { Telephonics } \\
\text { 236D 100-1 } \\
\text { HFA: } \\
\text { Sennheiser HD } \\
\text { 520 II }\end{array}$ & $\begin{array}{c}\text { Analyze results related to } \\
\text { high-frequency hearing } \\
\text { thresholds in individuals } \\
\text { aged } 18-29 \text { years without } \\
\text { otological problems. }\end{array}$ & $\begin{array}{l}\text { There were no significant } \\
\text { differences in hearing thresholds } \\
\text { between men and women aged } \\
\text { 18-29 years. Significant differences } \\
\text { in hearing thresholds between the } \\
\text { left and right ears were found only } \\
\text { at } 11-12 \mathrm{kHz} \text {. Over } 16 \mathrm{kHz} \text {, hearing } \\
\text { thresholds increased bilaterally. }\end{array}$ \\
\hline $\begin{array}{l}\text { Kumar et al., } \\
2016 \text { [19] }\end{array}$ & $\begin{array}{c}100 \\
30 \text { (10 men and } \\
20 \text { women), } 70 \text { people } \\
\text { using PLDs ( } 22 \text { men } \\
\text { and } 48 \text { women). } \\
\text { Study design } \\
\text { unknown. }\end{array}$ & $\begin{array}{l}15-30 \\
1 \text { age group. } \\
\text { Ears not distinguished. } \\
\text { Not divided by gender. }\end{array}$ & $\begin{array}{l}\text { New Delhi, } \\
\text { India }\end{array}$ & $\begin{array}{l}\text { CA } \\
0.125-8 \\
\text { HFA } \\
9-20\end{array}$ & $\begin{array}{c}\text { Labat } \\
\text { Audiolab } \\
\text { Audiometer }\end{array}$ & $\begin{array}{c}\text { CA/HFA: } \\
\text { Sennheiser HDA } \\
300\end{array}$ & $\begin{array}{l}\text { Examine changes in HFA } \\
\text { hearing thresholds in } \\
\text { PLD users and compare } \\
\text { them with an unexposed } \\
\text { group. }\end{array}$ & $\begin{array}{c}\text { Using a PLD for more than } 5 \text { years } \\
\text { at a high volume led to significantly } \\
\text { increased hearing thresholds at } 3, \\
10 \text { and } 13 \mathrm{kHz} \text {. }\end{array}$ \\
\hline $\begin{array}{l}\text { Le Prell et al., } \\
2013 \text { [20] }\end{array}$ & $\begin{array}{c}87 \\
\text { (34 men and } \\
53 \text { women) using PLDs. } \\
\text { Retrospective analysis. }\end{array}$ & $\begin{array}{l}\text { 18-31 } \\
1 \text { age group. } \\
\text { Left and right ears. } \\
\text { Divided by gender. }\end{array}$ & $\begin{array}{l}\text { Florida, } \\
\text { USA }\end{array}$ & $\begin{array}{l}\text { CA } \\
0.250-8 \\
\text { HFA } \\
10-16\end{array}$ & $\begin{array}{l}\text { Grason- } \\
\text { Stadler model } \\
61 \\
\text { (GSI 61) }\end{array}$ & $\begin{array}{l}\text { CA: } \\
\text { EAR 3A } \\
\text { HFA: } \\
\text { Sennheiser } \\
\text { HDA200 }\end{array}$ & $\begin{array}{c}\text { Determine whether HFA } \\
\text { thresholds for university } \\
\text { students differ } \\
\text { depending on exposure } \\
\text { to recreational noise. }\end{array}$ & $\begin{array}{l}\text { Subjects who used a PLD over the } \\
\text { long term ( } 5 \text { years or more) showed } \\
\text { statistically significant threshold } \\
\text { differences (3-6 dB higher) at the } \\
\text { highest frequencies tested } \\
(10-16 \mathrm{kHz}) .\end{array}$ \\
\hline
\end{tabular}


Table 2. Overview of the selected articles on hearing loss and high-frequency audiometry in populations exposed to workplace noise.

\begin{tabular}{|c|c|c|c|c|c|c|c|c|}
\hline $\begin{array}{l}\text { Author, } \\
\text { Year }\end{array}$ & $\begin{array}{l}\text { Number of } \\
\text { Respondents and } \\
\text { Study Design }\end{array}$ & $\begin{array}{c}\text { Age Range (Years) and } \\
\text { Groups }\end{array}$ & $\begin{array}{c}\text { City, } \\
\text { Country }\end{array}$ & $\begin{array}{l}\text { Audiometry } \\
\text { and Frequency } \\
\text { Range (kHz) }\end{array}$ & $\begin{array}{c}\text { Audiometer } \\
\text { Type }\end{array}$ & Headphones & Objective & Findings \\
\hline $\begin{array}{l}\text { Maccá et al., } \\
2014 \text { [27] }\end{array}$ & $\begin{array}{l}24 \text { EG ultrasound } \\
\text { (2 men and } 22 \text { women), } \\
113 \text { EG ( } 93 \text { men } \\
\text { and } 20 \text { women) } \\
148 \text { CG (62 men } \\
\text { and } 86 \text { women). } \\
\text { Study design } \\
\text { unknown. }\end{array}$ & $\begin{array}{c}\text { 15-59 } \\
\text { Age groups: 15-19, } \\
\text { 20-29, 30-39, 40-49, } \\
50-59 . \\
\text { Ears not } \\
\text { distinguished. } \\
\text { Not divided by gender. }\end{array}$ & $\begin{array}{l}\text { Padua, } \\
\text { Italy }\end{array}$ & $\begin{array}{l}\text { CA } \\
0.125-8 \\
\text { HFA } \\
9-18\end{array}$ & $\begin{array}{l}\text { Labat } \\
\text { Audiopack } \\
\text { audiometer }\end{array}$ & $\begin{array}{l}\text { CA: } \\
\text { Standard } \\
\text { headphones } \\
\text { HFA: } \\
\text { Sennheiser, } \\
\text { HD } 500\end{array}$ & $\begin{array}{l}\text { Investigate the effects of age, } \\
\text { ultrasound and noise on } \\
\text { high-frequency hearing } \\
\text { thresholds. }\end{array}$ & $\begin{array}{l}\text { After stratification for age, there was a } \\
\text { significantly higher hearing threshold in } \\
\text { EG than CG at } 9-10 \text { and } 14-15 \mathrm{kHz} \text { only } \\
\text { for those under } 30 \text { years of age. }\end{array}$ \\
\hline $\begin{array}{l}\text { Mehrparvar } \\
\text { et al., } \\
2014 \text { [22] }\end{array}$ & $\begin{array}{c}142 \mathrm{EG} \\
121 \mathrm{CG} \\
\text { Only men. } \\
\text { Cross-sectional, } \\
\text { prospective study. }\end{array}$ & $\begin{array}{l}\quad<50 \\
1 \text { age group. } \\
\text { Left and right ears. } \\
\text { Only men. }\end{array}$ & $\begin{array}{l}\text { City } \\
\text { unknown, } \\
\text { Iran }\end{array}$ & $\begin{array}{c}\text { CA } \\
0.5-8 \\
\text { HFA } \\
10-16\end{array}$ & $\begin{array}{l}\text { Interacoustic } \\
\text { AC40 }\end{array}$ & $\begin{array}{l}\text { CA: } \\
\text { TDH } 39 \\
\text { HFA. } \\
\text { Koss R/80 }\end{array}$ & $\begin{array}{l}\text { Compare three methods of } \\
\text { assessing hearing loss due to } \\
\text { noise (HFA, CA, DPOAE) }\end{array}$ & $\begin{array}{l}\text { The most commonly affected frequencies } \\
\text { with statistically significantly higher } \\
\text { hearing thresholds in EG compared with } \\
\text { CG were } 4 \text { and } 6 \mathrm{kHz} \text { in CA and } 14 \text { and } \\
16 \mathrm{kHz} \text { in HFA. HFA was the most } \\
\text { sensitive test for detection of hearing loss } \\
\text { in workers exposed to }>85 \mathrm{dBA} \text { noise. }\end{array}$ \\
\hline $\begin{array}{l}\text { Mehrparvar } \\
\text { et al., } \\
2011 \text { [28] }\end{array}$ & $\begin{array}{l}120 \text { EG (108 men } \\
\text { and } 12 \text { women) } \\
120 \text { CG (106 men } \\
\text { and } 14 \text { women). } \\
\text { Historic cohort. }\end{array}$ & $\begin{array}{l}<50 \\
1 \text { age group. } \\
\text { Left and right ears. } \\
\text { Not divided by gender. }\end{array}$ & $\begin{array}{l}\text { City } \\
\text { unknown, } \\
\text { Iran }\end{array}$ & $\begin{array}{l}\text { CA } \\
0.250-8 \\
\text { HFA } \\
10-16\end{array}$ & $\begin{array}{l}\text { Interacoustic } \\
\text { AC40 }\end{array}$ & $\begin{array}{l}\text { CA: } \\
\text { TDH } 39 \\
\text { HFA: } \\
\text { Koss R/80 }\end{array}$ & $\begin{array}{l}\text { Compare thresholds with } \\
\text { both CA and HFA in both } \\
\text { ears in exposed and } \\
\text { unexposed individuals to } \\
\text { assess the efficiency of the } \\
\text { methods when revealing } \\
\text { hearing loss. }\end{array}$ & $\begin{array}{l}\text { Statistically significantly higher mean } \\
\text { hearing thresholds in EG compared with } \\
\text { CG were found at } 4,6 \text { and } 16 \mathrm{kHz} \text {, with } \\
\text { the most significant differences found!at } \\
16 \mathrm{kHz} \text { in both!ears. }\end{array}$ \\
\hline $\begin{array}{l}\text { Ma et al., } \\
2018[23]\end{array}$ & $\begin{array}{c}134 \mathrm{EG} \\
101 \mathrm{CG} \\
\text { Only men. } \\
\text { Cross-sectional study. }\end{array}$ & $\begin{array}{c}20-59 \\
\text { Age groups: } \\
\text { 20-29, 30-39, } 40-49 \\
50-59 . \\
\text { Ears not } \\
\text { distinguished. Only } \\
\text { men. }\end{array}$ & $\begin{array}{l}\text { City } \\
\text { unknown, } \\
\text { China }\end{array}$ & $\begin{array}{l}\text { CA } \\
0.250-8 \\
\text { HFA } \\
9-20\end{array}$ & $\begin{array}{l}\text { Madsen } \\
\text { Conera }\end{array}$ & $\begin{array}{l}\text { CA: } \\
\text { TDH-39 } \\
\text { HFA: } \\
\text { Sennheiser } \\
\text { HDA!200 }\end{array}$ & $\begin{array}{l}\text { Investigate the usefulness of } \\
\text { HFA as an assessment test of } \\
\text { the hearing statuses of } \\
\text { civilian!pilots. }\end{array}$ & $\begin{array}{l}\text { Statistically significantly higher mean } \\
\text { hearing thresholds in EG compared with } \\
\text { CG were found at most of the high } \\
\text { frequencies tested. In particular, the } \\
\text { largest differences between hearing } \\
\text { thresholds were found at } 16 \mathrm{kHz} \text { for } \\
\text { subjects aged } 20-29 \text { and } 30-39 \text {, at } 12.5 \\
\mathrm{kHz} \text { for those aged } 40-49 \text { years old and at } \\
10 \mathrm{kHz} \text { for those aged } 50-59 \text { years old. }\end{array}$ \\
\hline $\begin{array}{l}\text { Ahmed } \\
\text { et al., } \\
2001[24]\end{array}$ & $\begin{array}{c}187 \mathrm{EG} \\
52 \mathrm{CG} \\
\text { Only men. } \\
\text { Cross-sectional study. }\end{array}$ & $\begin{array}{l}\text { Undefined-44 } \\
\text { Age groups: } \\
<25,25-34,35-44 . \\
\text { Ears not } \\
\text { distinguished. } \\
\text { Only men. }\end{array}$ & $\begin{array}{l}\text { City } \\
\text { unknown, } \\
\text { Saudi } \\
\text { Arabia }\end{array}$ & $\begin{array}{c}\text { CA } \\
0.250-8 \\
\text { HFA } \\
10-18\end{array}$ & $\begin{array}{l}\text { Interacoustics } \\
\text { AS10HF }\end{array}$ & $\begin{array}{c}\text { CA: } \\
\text { Koss HV-1A } \\
\text { HFA: } \\
\text { TDH-50P }\end{array}$ & $\begin{array}{l}\text { Investigate the reliability and } \\
\text { effects of age and noise on } \\
\text { HFA hearing thresholds. }\end{array}$ & $\begin{array}{l}\text { A multivariate analysis showed that the } \\
\text { primary indicator of the hearing } \\
\text { threshold at high frequencies is age, and } \\
\text { noise exposure is a secondary predictor of } \\
\text { hearing thresholds at high frequencies } \\
(10-18 \mathrm{kHz}) .\end{array}$ \\
\hline
\end{tabular}


Table 2. Cont.

\begin{tabular}{|c|c|c|c|c|c|c|c|c|}
\hline $\begin{array}{l}\text { Author, } \\
\text { Year }\end{array}$ & $\begin{array}{l}\text { Number of } \\
\text { Respondents and } \\
\text { Study Design }\end{array}$ & $\begin{array}{c}\text { Age Range (Years) and } \\
\text { Groups }\end{array}$ & $\begin{array}{l}\text { City, } \\
\text { Country }\end{array}$ & $\begin{array}{l}\text { Audiometry } \\
\text { and Frequency } \\
\text { Range (kHz) }\end{array}$ & $\begin{array}{l}\text { Audiometer } \\
\text { Type }\end{array}$ & Headphones & Objective & Findings \\
\hline $\begin{array}{l}\text { Somma } \\
\text { et al., } \\
2008 \text { [25] }\end{array}$ & $\begin{array}{c}84 \text { EG } \\
98 \text { CG } \\
\text { Only men. } \\
\text { Study design } \\
\text { unknown. }\end{array}$ & $\begin{array}{c}21-60 \\
\text { Age groups: } 21-30 \\
\text { 31-40, } 41-50,51-60 . \\
\text { Ears not } \\
\text { distinguished. Only } \\
\text { men. }\end{array}$ & $\begin{array}{l}\text { City } \\
\text { unknown, } \\
\text { Italy }\end{array}$ & $\begin{array}{l}\text { CA } \\
0.250-8 \\
\text { HFA } \\
9-18\end{array}$ & $\begin{array}{c}\text { Amplaid A319, } \\
\text { Amplifon }\end{array}$ & $\begin{array}{l}\text { CA: } \\
\text { TDH-49 } \\
\text { HFA: } \\
\text { Sennheiser } \\
\text { HDA!200 }\end{array}$ & $\begin{array}{l}\text { Compare HFA and CA to } \\
\text { assess thresholds among } \\
\text { workers exposed to } \\
\text { workplace noise. }\end{array}$ & $\begin{array}{l}\text { Statistically significantly higher hearing } \\
\text { thresholds between EG and CG were } \\
\text { found for those aged } 21-30 \text { years old at } \\
\text { all frequencies (9-18 kHz) and for those } \\
\text { aged 31-40 years old at frequencies of } \\
9-14 \mathrm{kHz} \text {. }\end{array}$ \\
\hline $\begin{array}{l}\text { Korres et al., } \\
2008[29]\end{array}$ & $\begin{array}{l}139 \text { EG ( } 68 \text { men and } \\
53 \text { women) } \\
32 \text { CG ( } 18 \text { men and } \\
14 \text { women). } \\
\text { Study design } \\
\text { unknown. }\end{array}$ & $\begin{array}{c}24-55 \\
1 \text { age group. } \\
\text { Left and right ears. } \\
\text { Not divided by gender. }\end{array}$ & $\begin{array}{l}\text { City } \\
\text { unknown, } \\
\text { Greece }\end{array}$ & $\begin{array}{l}\text { CA } \\
0.250-8 \\
\text { HFA } \\
9-20\end{array}$ & $\begin{array}{l}\text { Amplaid 321, } \\
\text { Twinchannel }\end{array}$ & $\begin{array}{l}\text { CA: } \\
\text { TDH-49 } \\
\text { HFA: } \\
\text { Sennheiser } \\
\text { HDA 200 }\end{array}$ & $\begin{array}{c}\text { Evaluate hearing in } \\
\text { industrial workers exposed } \\
\text { to workplace noise using CA } \\
\text { and HFA and compare it } \\
\text { with CG. }\end{array}$ & $\begin{array}{l}\text { Statistically significantly higher hearing } \\
\text { thresholds between EG and CG were } \\
\text { found at } 4-18 \mathrm{kHz} \text {, especially at } 12.5-18 \\
\mathrm{kHz} \text {. A statistically significant } \\
\text { correlation between an increased } \\
\text { duration of exposure and higher } \\
\text { hearing thresholds was found at all } \\
\text { frequencies except for } 10 \mathrm{kHz} \text {. }\end{array}$ \\
\hline $\begin{array}{l}\text { Rocha et al., } \\
2010[26]\end{array}$ & $\begin{array}{l}47 \text { EG } \\
33 \text { CG } \\
\text { Only men. } \\
\text { Cross-sectional, } \\
\text { retrospective } \\
\text { cohort study. }\end{array}$ & $\begin{array}{l}\text { 30-49 } \\
\text { Age groups: } \\
\text { 30-39, } 40-49 . \\
\text { Ears not } \\
\text { distinguished. } \\
\text { Only men. }\end{array}$ & $\begin{array}{l}\text { Rio de } \\
\text { Janeiro, } \\
\text { Brazil }\end{array}$ & $\begin{array}{l}\text { CA } \\
0.250-8 \\
\text { HFA } \\
9-20\end{array}$ & $\begin{array}{l}\text { Interacoustic } \\
\quad \mathrm{AC} 40\end{array}$ & $\begin{array}{c}\text { CA: } \\
\text { TDH-39P } \\
\text { HFA: } \\
\text { Koss HV /PRO }\end{array}$ & $\begin{array}{l}\text { Analysis of HFA results in } \\
\text { people exposed to noise with } \\
\text { normal results for CA. }\end{array}$ & $\begin{array}{l}\text { The EG had a statistically significantly } \\
\text { higher hearing threshold than CG at } \\
16 ! \mathrm{kHz} \text { in participants aged } 30-39 \text { years. } \\
\text { The results were most significant in the } \\
40-49 \text { years age group, where EG } \\
\text { showed significantly higher hearing } \\
\text { thresholds than CG at } 14 \text { and } 16 \mathrm{kHz} \text {. }\end{array}$ \\
\hline $\begin{array}{l}\text { Goncalves } \\
\text { et al., } \\
2015 \text { [30] }\end{array}$ & $\begin{array}{l}40 \text { EG ( } 10 \text { men and } \\
32 \text { women }) \\
\text { CG } 40 \\
\text { Historic cohort study. }\end{array}$ & $\begin{array}{l}\text { 23-61 } \\
1 \text { age group. } \\
\text { Left and right ears. } \\
\text { Divided by gender. }\end{array}$ & $\begin{array}{l}\text { Curitiba, } \\
\text { Brazil }\end{array}$ & $\begin{array}{l}\text { CA } \\
0.5-8 \\
\text { HFA } \\
9-16\end{array}$ & $\begin{array}{l}\text { Madsen } \\
\text { Itera II, GN } \\
\text { Otometrics }\end{array}$ & $\begin{array}{l}\text { CA: } \\
\text { Standard } \\
\text { HFA: } \\
\text { Sennheiser } \\
\text { HDA 200 }\end{array}$ & $\begin{array}{l}\text { Use HFA to evaluate hearing } \\
\text { among dentists exposed to } \\
\text { workplace noise for varying } \\
\text { durations. }\end{array}$ & $\begin{array}{l}\text { Statistically significantly higher hearing } \\
\text { thresholds in EG compared with CG } \\
\text { were observed at } 0.5,1,6 \text { and } 8 \mathrm{kHz} \text { in } \\
\text { the right ear. No differences were } \\
\text { observed between the EG and CG for } \\
\text { high frequencies. }\end{array}$ \\
\hline $\begin{array}{l}\text { Antonioli } \\
\text { et al., } \\
2016 \text { [31] }\end{array}$ & $\begin{array}{l}\text { Exposed workers and } \\
\text { unexposed people. } \\
\text { Both genders. } \\
\text { Systematic review, } \\
\text { meta-analysis. }\end{array}$ & $\begin{array}{c}18-60 \\
\text { Different age groups. } \\
\text { Ears not } \\
\text { distinguished. } \\
\text { Not divided by gender. } \\
6 \text { studies }\end{array}$ & $\begin{array}{l}\text { Many } \\
\text { countries }\end{array}$ & $\begin{array}{l}\text { CA } \\
0.250-8 \\
\text { HFA } \\
10-20\end{array}$ & $\begin{array}{l}\text { Interacoustics } \\
\text { AS10HF; } \\
\text { Amplaid A3 } \\
\text { 19; Amplaid } \\
\text { A321; } \\
\text { Interacoustic } \\
\text { AC!40; } \\
\text { Siemens SD50; } \\
\text { GSI!61 }\end{array}$ & $\begin{array}{c}\text { HFA: } \\
\text { Koss R/80; } \\
\text { HDA200; } \\
\text { R80; HDA200; } \\
\text { HD 200; } \\
\text { TDH-39p }\end{array}$ & $\begin{array}{l}\text { Retrospective and secondary } \\
\text { systematic revision of } \\
\text { publications using HFA to } \\
\text { monitor the hearing of } \\
\text { workers exposed to } \\
\text { workplace noise. }\end{array}$ & $\begin{array}{l}\text { At } 16 \mathrm{kHz}, \mathrm{HFA} \text { is sensitive enough for } \\
\text { the early detection of hearing loss. This } \\
\text { is true for } 4 \mathrm{kHz} \text { as well, but the } \\
\text { outcome is not as significant. Further } \\
\text { studies are therefore needed to confirm } \\
\text { the importance of HFA for the early } \\
\text { detection of hearing loss in people } \\
\text { exposed to workplace noise. }\end{array}$ \\
\hline
\end{tabular}

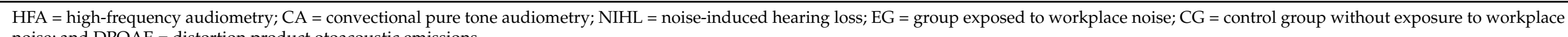
noise; and DPOAE = distortion product otoacoustic emissions. 
As shown in Table 2, studies in which people were exposed to noise in the workplace involved 40-282 subjects. The control groups had 32-142 participants. Five of the studies involving workplace noise exposure only assessed men [22-26], four of them included both men and women [27-30], and one study was a review [31]. The age range was 15-61 years, and the age groups used also varied. All studies compared CA and HFA, and five of them even included a frequency of $20 \mathrm{kHz}$. An Interacoustic audiometer was used in four studies; three of these used the AC 40 type [22,26,28], and one used the AS10HF type [31]. Two studies used a Madsen audiometer [23,30], a further two used the Amplaid [22,28], and one study used a Labat Audiopack [27]. Sennheiser headphones were used in five studies; four of them used the HDA 200 [23-25,30], and one used the HDA 500 [27]. Three studies used Koss headphones [22,26,28]. The systematic review conducted by Antonioli et al. compared six studies with six different audiometers and different types of headphones [31].

\subsection{Studies with People Not Exposed to Workplace Noise}

Articles monitoring high-frequency hearing loss in unexposed individuals reported an increase in hearing thresholds with an increasing frequency as well as with increasing age $[17,18,21]$. In populations unexposed to workplace noise, significantly higher hearing thresholds were found at frequencies of 14 and $16 \mathrm{kHz}$, and these increased with age [17,20]. In a representative cohort of 645 people, it was found that those under 40 years were able detect sounds at frequencies of up to $18 \mathrm{kHz}$, people between 40 and 49 could detect sounds at frequencies of up to $14 \mathrm{kHz}$, and those older than 50 could only detect sounds at frequencies of up to $11.2 \mathrm{kHz}$. The mean hearing thresholds at each frequency $(11.2$ to $20 \mathrm{kHz})$ were lower in women than men. Statistically significantly higher hearing thresholds were found in men than in women at 12.5 and $16 \mathrm{kHz}$ in the $20-29$ year group, at $16 \mathrm{kHz}$ in the 30-39 year group, at 11.2 and $16 \mathrm{kHz}$ in the 40-49 year group, at $10 \mathrm{kHz}$ in the 50-59 year group and at 12.5 and $18 \mathrm{kHz}$ in the 60-69 year group. Nevertheless, a comparison of different studies showed that the hearing thresholds for men and women were similar [17]. This was confirmed by Barbosa de Sá et al., who found no significant difference in hearing thresholds between men and women aged 18-29 years [18]. Oppitz et al. found higher hearing threshold values in the right ear than in the left ear at 10, 11 and $14 \mathrm{kHz}$. At frequencies above $14 \mathrm{kHz}$, the progressive increase was proportional to the frequency and was bilateral (binaural), with threshold values increasing with age at all frequencies [21]. Barbosa de Sá et al. found that the thresholds were similar in the left and right ears, with significant differences between the ears only being observed at 11 and $12 \mathrm{kHz}$, with the right ear being worse [18].

Kumar et al. found that high frequency thresholds could be used for the early detection of noise-induced hearing loss (NIHL) in PLD users. It was found that those using PLDs at high volumes for less than 5 years showed no significant differences in hearing threshold values. In contrast, using a PLD for more than 5 years led to significantly increased hearing thresholds at 3, 10 and $13 \mathrm{kHz}$ [14]. Le Prell et al. found statistically significant threshold differences (3-6 dB higher) at $10-16 \mathrm{kHz}$ in respondents who had used a PLD for a long period of time or at a very high volume after just 5 years [20]. HFA could be used for the early detection of NIHL in PLD users $[19,20]$. Both studies examined the age category of below 31 years. This generation generally has more frequent usage of mobile phones, headphones and other PLDs.

Specific threshold analyses focused on risk stratification of permanent hearing loss according to clearly defined levels of exposure to music through PLDs are missing. This is due to many restrictions of conducting such research that are related to the long latency time from exposure to effect, the problems to correctly estimate the exposure and the lack of sensitive measures to detect early signs of hearing loss [32].

\subsection{Studies on Workplace Noise Exposure}

Age was found to be the primary predictor and noise exposure the secondary predictor of an increase in high-frequency hearing thresholds. The results suggest that HFA may be 
useful for the early diagnosis of noise-induced hearing loss, especially for younger groups of workers (up to 30 years of age) [22-30].

HFA is more sensitive for NIHL detection than CA; it can be used for early diagnosis of hearing loss, and thus it can contribute to the prevention of hearing loss, even at lower frequencies, especially at frequencies used for speech $[23,25,28]$. Studies comparing the EG and the CG found that in those aged over 30 years, hearing loss was apparent in HFA before it became apparent in CA $[23,25,27]$. Studies suggest that HFA rather than CA could be useful as an early probe for hearing loss resulting from noise [24]. In a sample of working age respondents, Korres et al. showed that HFA is a useful addition to CA for the audiological evaluation of people exposed to workplace noise [29]. Mehrparvar et al. compared the following methods: $\mathrm{CA}$, distortion product otoacoustic emissions and HFA. HFA was confirmed as the most sensitive method for detecting hearing loss from hazardous noise exposure in the workplace [22]. Maccá et al. stated that hearing loss at high frequencies is affected not only by age, but also by the duration of exposure to a noisy work environment [27]. This was confirmed by Goncalves et al., who showed that dentists working in surgeries for more than 10 years had significantly greater hearing loss at high frequencies compared with those in the control group. Eighty-one percent of dentists were not informed of the risk of noise during their university studies, and $15 \%$ of dentists (EG) had sensorineural hearing impairment, while in the CG, the frequency of occurrence was $2.5 \%$ [30]. Korres et al. found a statistically significant correlation between the duration of exposure and the hearing threshold at all frequencies [29]. Changes in high-frequency thresholds were found to be accelerated by noise exposure in the first few years, suggesting that HFA could be a useful preventative measure for monitoring younger workers exposed to workplace noise [25]. Statistically significant differences were found between people exposed to noise for $<10$ years and a control group at $2-8 \mathrm{kHz}, 9-10 \mathrm{kHz}$ and $14-15 \mathrm{kHz}$ in people aged up to 39 years of age. Age is a secondary factor to hearing loss; at conventional frequencies, the hearing threshold increases after the age of 20. After stratification for age, significant differences between the EG and the CG were found at $9-10 \mathrm{kHz}$ and $14-15 \mathrm{kHz}$ only for those who were aged below 30 years [27]. Larger differences were found between the exposed and control groups at $4-18 \mathrm{kHz}$, and these were more evident at $12.5-18 \mathrm{kHz}$. There was a statistically significant correlation between the differences in thresholds and duration of exposure at all frequencies $(0.25-20 \mathrm{kHz})$, except for $10 \mathrm{kHz}$ [29]. HFA performs well in the frequency range of $12.5-18 \mathrm{kHz}$, but there is greater variability in the results compared with CA [29]. The results of Rocha et al. were the most significant for the 40-49-year age group, and the exposed group showed significantly higher thresholds than the control group at 14 and $16 \mathrm{kHz}$. The EG of individuals aged 30-39 years showed a significantly higher threshold than CG only at $16 \mathrm{kHz}$ [26]. According to Ma et al., the most frequently affected frequencies for subjects aged 30-39 years are 14 and $16 \mathrm{kHz}$ [23]. A stepwise regression analysis showed that in 21-40-year-old workers, the effect of noise was apparent with both CA and HFA, while in older people, the noise effect was only apparent at frequencies of up to $6 \mathrm{kHz}$, and the impact of age was significantly higher at higher frequencies [25]. Mehrparvar et al. stated that the most frequently affected frequencies were 4 and $6 \mathrm{kHz}$ for $\mathrm{CA}$ and 14 and $16 \mathrm{kHz}$ for HFA [22]. They also found abnormal hearing in $29 \%$ of participants exposed to workplace noise with CA, $69 \%$ with HFA, $22 \%$ with low-frequency DPOAE and 52\% with high-frequency DPOAE [22].

In another study comparing the EG and the CG, Mehrparvar et al. found that in the EG, the highest hearing thresholds were observed at $4 \mathrm{kHz}$ in the left ear, $6 \mathrm{kHz}$ in the right ear and $16 \mathrm{kHz}$ when both ears were tested. The hearing threshold was significantly higher at $16 \mathrm{kHz}$ than at $4 \mathrm{kHz}$. There was no statistically significant difference between the right and left ears in either group. Hearing loss was more common in males than females, but the difference was not statistically significant [28]. Ma et al. found differences in thresholds between the EG and the CG in all age categories [23]. Compared with the $\mathrm{CG}$, the mean threshold at frequencies of $0.25-20 \mathrm{kHz}$ in the EG increased by $3.15 \mathrm{~dB}$ for CA and by $7.8 \mathrm{~dB}$, respectively, for HFA for the 20-29-year age group; by 2.4 and $9.9 \mathrm{~dB}$, 
respectively, for the 30-39-year age group; by 3.8 and $8.2 \mathrm{~dB}$, respectively, for the 40-49year age group; and by 10.8 and $16.9 \mathrm{~dB}$, respectively, for the 50-59-year age group [23]. Additionally, Ma et al. found that the results for particular frequencies were more sensitive to noise than other frequencies: 14 and $16 \mathrm{kHz}$ for the 20-29-year age group, 11.2 and $12.5 \mathrm{kHz}$ for the 40-49-year age group and 11.2 and $10 \mathrm{kHz}$ for the 50-59-year age group. Significant differences in HFA were also observed between the EG and the CG with normal CA hearing thresholds. Seventy-five percent of pilots were shown to have hearing loss in at least one ear and at least one frequency with CA; the corresponding proportion was $95 \%$ with HFA [23].

Statistically significantly higher hearing thresholds in the EG than the CG were found at all frequencies (9-18 kHz) for the 21-30-year age group and at frequencies of 9-14 kHz for those aged 31-40 years old. On the other hand, in the 41-50-year age group, statistically significantly higher hearing thresholds were found in the EG compared with the CG only at 12.5 and $14 \mathrm{kHz}$, while no significant differences were found for the oldest workers aged 51-60 years old [25].

Antonioli et al. selected over 6000 articles for a retrospective and systematic review of the use of HFA for the monitoring of hearing loss of those exposed to workplace noise [31]. Of these, only six articles met the criteria for the study, and the difference between exposed and unexposed persons was seen mainly at $16 \mathrm{kHz}$, with a slightly smaller difference at $4 \mathrm{kHz}$. Antonioli et al. conducted a meta-analysis that clearly determined the hearing threshold for each frequency (i.e., 2, 3, 4, 6, 8, 10, 14 and $16 \mathrm{kHz}$ ). They evaluated the mean, standard deviation, median and $95 \%$ CI for the EG and the CG, but they did not take age groups into account, and these differed among the studies. The authors are themselves critical of these results and recommend the establishment of a uniform methodology for HFA testing with regard to age groups, comorbidities, hearing loss, gender and both occupational and leisure noise exposure. In terms of occupational exposure, information on the use of personal protective equipment should be included. The meta-analysis suggests that at $16 \mathrm{kHz}, \mathrm{HFA}$ is sensitive enough to identify early hearing loss in the CG. This is true for $4 \mathrm{kHz}$ as well, but the outcome is not as significant. Further studies are therefore needed to validate the importance of using HFA to monitor early hearing loss in people exposed to workplace noise [31].

Our review compared the use of HFA in populations exposed to workplace noise in addition to comparing the EG and CG with regard to age categories, taking into account both CA and HFA. We found significant differences in the study methodologies used for HFA, mainly in terms of age group divisions. Some of the studies investigated did not consider particular age groups and evaluated hearing thresholds in HFA for one single group, regardless of the ages of the included individuals [18-20,22,28-31]. Previous studies have confirmed that HFA detects deterioration in the hearing threshold with an increasing age, increasing frequency and with noise exposure at younger ages compared with CA. A cross-sectional study of 6451 individuals with a mean age of $59 \pm 4$ years designed to be representative of the US population found a decrease in cognition with every $10 \mathrm{~dB}$ reduction in hearing, which continued below the clinical threshold such that subclinical levels of hearing impairment (below $25 \mathrm{~dB}$ ) were significantly related to lower cognition $[4,31]$. Therefore, CA studies aimed at finding people with hearing loss should be extended to HFA studies with the same design.

\subsection{Limitations}

The limitations of this review article are as follows:

1. Only a few published studies have used HFA to determine hearing thresholds in an unexposed population, and even fewer studies have compared cohorts exposed and unexposed to workplace noise;

2. The different age groups considered in the existing studies have varying age ranges. Some studies have reported hearing thresholds independently of age, even though the hearing threshold worsens with age at each frequency; 
3. The standards for hearing loss classification and their corresponding audiometry values have not yet been defined for frequencies other than $0.125-8 \mathrm{kHz}$.

Due to these restrictions, it was not possible to perform a meta-analysis of the data. We could not compare the results of the individual studies with normative data. HFA needs to be studied further, especially with regard to dependence on age and other variables.

\section{Conclusions}

Until recently, it was not straightforward to examine hearing thresholds with HFA. HFA has made it into clinical practice mainly due to recent developments in the technology and the construction of special headphones and specially adapted audiometers. Although it can be used for the early detection of hearing loss, normative data (i.e., boundary values that indicate an as-yet unimpaired hearing threshold) have not yet been established. The effect of NIHL first became apparent through the use of HFA rather than CA. NIHL is a risk factor that is easily preventable in working environments, and moreover, it is highly underestimated as a risk factor in non-working environments in ordinary human life. Among people unexposed to workplace noise, significantly higher hearing thresholds are found with increasing age. The ability to detect sound is up to $18 \mathrm{kHz}$ in those aged under 40 years, while it decreases to $14 \mathrm{kHz}$ in the $40-49$-year age group, and those above 50 years are able to detect sound only up to $11.2 \mathrm{kHz}$. Significantly higher hearing thresholds in EG compared with CG have been found in the $9-18 \mathrm{kHz}$ range, especially at $16 \mathrm{kHz}$. Previous studies have also found higher hearing thresholds at $10-16 \mathrm{kHz}$ in respondents aged under 31 years who have used PLDs for longer than 5 years. It is obvious that PLD users in younger age categories require more attention in terms of hearing loss detection, since these age categories are more familiar with modern technologies, including different types of PLDs.

The hearing threshold increases with increasing age, frequency and noise exposure, and this is detectable earlier when HFA rather than CA is used.

A number of studies on HFA have been conducted, but each had a different design, and so they cannot be reliably compared to other studies. A harmonized methodology needs to be established that takes into account variables such as age (e.g., WHO age categories), sex, comorbidities and noise exposure, as well as other socioeconomic and psychosocial factors and the usage of PLDs. This is necessary given the broader objective of earlier detection of hearing loss and compensation for this impairment. According to this context and the development of modern technologies, HFA should be added into the standard examination protocol for the early detection of hearing loss.

Author Contributions: Conceptualization of the study, M.Š.; data selection and extraction, M.Š.; analysis, M.Š., E.M. and M.K.; writing-original draft preparation, M.Š., E.M. and M.K.; review and editing, E.M. and M.K.; supervision M.Š., E.M. and M.K. All authors have read and agreed to the published version of the manuscript.

Funding: This research was funded by the Technology Agency of the Czech Republic (No. TJ04000059: "Quality of life improvement by early detection of hearing loss using high-frequency audiometry").

Institutional Review Board Statement: Not applicable.

Informed Consent Statement: Not applicable.

Data Availability Statement: The study materials and the details of all analyses are available from the corresponding authors upon reasonable request.

Acknowledgments: The authors are grateful to the Technology Agency of the Czech Republic.

Conflicts of Interest: The authors declare no conflict of interest. 


\section{References}

1. World Health Organization. International Classification of Functioning, Disability and Health; World Health Organization: Geneva, Switzerland, 2001; Available online: https:/ / www.who.int/classifications/icf/en/ (accessed on 20 February 2021).

2. World Health Organization. Addressing the Rising Prevalence of Hearing Loss. 2018. Available online: https://apps.who.int/iris/ bitstream/handle/10665/260336/9789241550260-eng.pdf?fbclid=IwAR0tQbo1bvObm8h5_hD0PBPmfPowiHLiQIDAJqJcnV1 ThYnDy1fnb-q9flc (accessed on 20 February 2021).

3. World Health Organization. Deafness and Hearing Loss. 2020. Available online: https://www.who.int/news-room/fact-sheets/ detail/deafness-and-hearing-loss (accessed on 20 February 2021).

4. Livingston, G.; Huntley, J.; Sommerlad, A.; Ames, D.; Ballard, C.; Banerjee, S.; Brayne, C.; Burns, A.; Cohen-Mansfield, J.; Cooper, C.; et al. Dementia prevention, intervention, and care: 2020 report of the Lancet Commission. Lancet 2020, 396, 413-446. [CrossRef] [PubMed]

5. Hear-It AISBL. Evaluation of the Social and Economic Costs of Hearing Impairment. 2019. Available online: https://www.hearit.org/sites/default/files/2021-01/Hear-it\%20Report\%20Hearing\%20Loss\%20Numbers\%20and\%20Costs\%202019.pdf (accessed on 20 February 2021).

6. Wong, A.C.Y.; Froud, K.E.; Hsieh, Y.S.Y. Noise-induced hearing loss in the 21st century: A research and translational update. World J. Otorhinolaryngol. 2013, 3, 58-70. [CrossRef]

7. World Health Organization. Age-Related Hearing Loss (Presbycusis). 2013. Available online: https://www.who.int/news-room/ q-a-detail/deafness-and-hearing-loss-age-related-hearing-loss-(presbycusis) (accessed on 20 February 2021).

8. Cunningham, L.L.; Tucci, D.L. Hearing Loss in Adults. N. Engl. J. Med. 2017, 377, 2465-2473. [CrossRef]

9. Mick, P.; Kawachi, I.; Lin, F.R. The Association between Hearing Loss and Social Isolation in Older Adults. Otolaryngol. Neck Surg. 2013, 150, 378-384. [CrossRef]

10. Clark, W.; Ohlemiller, K.K. Anatomy and Physiology of Hearing for Audiologist, 1st ed.; Thomson Delmar: Clifton Park, NY, USA, 2008; pp. 1-458.

11. Ackley, R.S.; Decker, T.N.; Limb, C.H.J. An Essential Guide to Hearing and Balance Disorders; Psychology Press: East Sussex, UK, 2007; pp. 1-504.

12. World Health Organization. Hearing Measurement. 2001. Available online: https://www.who.int/occupational_health/ publications/noise8.pdf (accessed on 20 February 2021).

13. International Electrotechnical Commission. Electroacoustics-Audiometric Equipment_-Part 1: Equipment for Pure-Tone and Speech Audiometry; IEC 60645-1; European Committee for Electrotechnical Standardization: Brussels, Belgium, 2017.

14. International Organization for Standardization. Acoustics-Audiometric Test Methods: Part 1: Pure-Tone Air and Bone Con-duction Audiometry; ISO 8523-1; International Organization of Standards: Geneva, Switzerland, 2010.

15. International Organization for Standardization. Acoustics—Preferred Frequencies; ISO 266; International Organization of Standards: Geneva, Switzerland, 1997.

16. Couth, S.; Prendergast, G.; Guest, H.; Munro, K.J.; Moore, D.R.; Plack, C.J.; Ginsborg, J.; Dawes, P. Investigating the effects of noise exposure on self-report, behavioral and electrophysiological indices of hearing damage in musicians with normal audiometric thresholds. Hear. Res. 2020, 395, 108021. [CrossRef]

17. Valiente, A.R.; Trinidad, A.; Berrocal, J.R.G.; Górriz, C.; Camacho, R.R. Extended high-frequency (9-20 kHz) audiometry reference thresholds in 645 healthy subjects. Int. J. Audiol. 2014, 53, 531-545. [CrossRef]

18. Barbosa de Sá, L.C.; Melo Tavares de Lima, M.C.; Tomota, S.; Monte Coelho Frota, S.M.; Aquino Santos, G.; Rodrigues Garcia, T. Analisys of high frequency auditory thresholds in individuals aged between 18 and 29 years with no ontological complaints. Rev. Bras. Otorrinolaringol. 2007, 73, 215-225.

19. Kumar, P.; Upadhyay, P.; Kumar, A.; Kumar, S.; Singh, G.B. Extended high frequency audiometry in users of personal listening devices. Am. J. Otolaryngol. 2017, 38, 163-167. [CrossRef] [PubMed]

20. Le Prell, C.G.; Spankovich, C.; Lobariñas, E.; Griffiths, S.K. Extended High-Frequency Thresholds in College Students: Effects of Music Player Use and Other Recreational Noise. J. Am. Acad. Audiol. 2013, 24, 725-739. [CrossRef]

21. Oppitz, S.J.; Da Silva, L.C.L.; Garcia, M.V.; Da Silveira, A.F. Limiares de audibilidade de altas frequências em indivíduos adultos normo-ouvintes. CoDAS 2018, 30. [CrossRef] [PubMed]

22. Mehrparvar, A.H.; Mirmohammadi, S.J.; Davari, M.H.; Mostaghaci, M.; Mollasadeghi, A.; Bahaloo, M.; Hashemi, S.H. Conventional Audiometry, Extended High-Frequency Audiometry, and DPOAE for Early Diagnosis of NIHL. Iran. Red Crescent Med. J. 2014, 16, e9628. [CrossRef] [PubMed]

23. Ma, F.; Gong, S.; Liu, S.; Hu, M.; Qin, C.; Bai, Y. Extended High-Frequency Audiometry (9-20 kHz) in Civilian Pilots. Aerosp. Med. Hum. Perform. 2018, 89, 593-600. [CrossRef]

24. Ahmed, H.O.; Dennis, J.H.; Badran, O.; Ismail, M.; Ballal, S.G.; Ashoor, A.; Jerwood, D. High-frequency (10-18 kHz) hearing thresholds: Reliability, and effects of age and occupational noise exposure. Occup. Med. 2001, 51, 245-258. [CrossRef]

25. Somma, G.; Pietroiusti, A.; Magrini, A.; Coppeta, L.; Ancona, C.; Gardi, S.; Messina, M.; Bergamaschi, A. Extended high-frequency audiometry and noise induced hearing loss in cement workers. Am. J. Ind. Med. 2008, 51, 452-462. [CrossRef] [PubMed]

26. Da Rocha, R.L.O.; Atherino, C.C.T.; Frota, S.M.M.C. Audiometria de altas frequências em bombeiros militares com audiometria normal expostos ao ruído. Braz. J. Otorhinolaryngol. 2010, 76, 687-694. [CrossRef] 
27. Maccà, I.; Scapellato, M.L.; Carrieri, M.; Maso, S.; Trevisan, A.; Bartolucci, G.B. High-frequency hearing thresholds: Effects of age, occupational ultrasound and noise exposure. Int. Arch. Occup. Environ. Health 2014, 88, 197-211. [CrossRef] [PubMed]

28. Mehrparvar, A.H.; Ghoreyshi, A.; Loukzadeh, Z.; Mirmohammadi, S.J.; Mollasadeghi, A. High-frequency audiometry: A means for early diagnosis of noise-induced hearing loss. Noise Health 2011, 13, 402-406. [CrossRef] [PubMed]

29. Korres, G.S.; Balatsouras, D.G.; Tzagaroulakis, A.; Kandiloros, D.; Ferekidis, E. Extended high-frequency audiometry in subjects exposed to occupational noise. B-ENT 2008, 4, 147-155.

30. Santos, L.; Lobato, D.; Ribas, A.; Lacerda, A.B.M.; Marques, J.; Gonçalves, C.G.D.O. Characterization of Hearing Thresholds from 500 to 16,000 Hz in Dentists: A Comparative Study. Int. Arch. Otorhinolaryngol. 2014, 19, 156-160. [CrossRef]

31. Momensohn-Santos, T.M.; Benaglia, T.A.S.; Antonioli, C.A.S. High-frequency Audiometry Hearing on Monitoring of Individuals Exposed to Occupational Noise: A Systematic Review. Int. Arch. Otorhinolaryngol. 2015, 20, 281-289. [CrossRef]

32. Śliwińska-Kowalska, M.; Zaborowski, K. WHO Environmental Noise Guidelines for the European Region: A Systematic Review on Environmental Noise and Permanent Hearing Loss and Tinnitus. Int. J. Environ. Res. Public Health 2017, 14, 1139. [CrossRef] [PubMed] 